\title{
A case of a hepatosplenomesenteric trunk combined with a hepatocolic trunk
}

\author{
Sarah E. Johnson, David D. Odineal, Amy E. Steele, Valerie M. Stone, Richard P. Tucker \\ Department of Cell Biology and Human Anatomy, University of California at Davis, Davis, California, USA.
}

\begin{abstract}
An understanding of the variations in the blood supply of the foregut and midgut are of critical importance to surgeons performing transplants, liver and biliary surgery, resection of tumors and various gastrointestinal procedures, as well as to interventional radiologists engaged in vessel embolization. During the dissection of a 95-year-old female cadaver as part of a course in medical gross anatomy at the University of California at Davis a rare series of vascular variations were observed. The left gastric artery arose independently from the abdominal aorta at the location of a typical celiac trunk. The common hepatic artery and splenic artery branched from a common vessel originating from a hepatosplenomesenteric trunk. Just inferior to the hepatosplenic trunk a hepatocolic trunk, which gave rise to an accessory right hepatic artery, dorsal pancreatic artery and a wandering mesenteric artery, branched from the superior mesenteric artery. This rare combination of clinically relevant variations was likely due to the abnormal partitioning and regression of the primitive splanchnic arteries during embryonic development.
\end{abstract}

Keywords: anatomical variation; arc of Riolan; celiac trunk; celiacomesenteric trunk; superior mesenteric artery

Anatomy 2017;11(1):46-49 @2017 Turkish Society of Anatomy and Clinical Anatomy (TSACA)

\section{Introduction}

The celiac trunk is a large, unpaired vessel that arises from the abdominal aorta just inferior to the aortic hiatus. It is typically tripartite in arrangement, giving rise to the left gastric artery, splenic artery and common hepatic artery. ${ }^{[1]}$ The superior mesenteric artery, which is a source of jejunal and iliac branches, arises from the anterior surface of the abdominal aorta approximately $1 \mathrm{~cm}$ inferior to the celiac trunk. Variations in this arrangement are common, well documented, and have clinical relevance to surgeons and radiologists. For example, Chen et al ${ }^{[2]}$ found that 875 of 974 cadavers $(89.8 \%$ ) dissected at the Gifu University School of Medicine between 1980 and 2007 had a typical tripartite celiac trunk arising independently from the superior mesenteric artery. According to the classification system introduced by Adachi (as cited by Chen et al. ${ }^{[2]}$ ) this typical pattern is referred to as Type I. The most common variation is characterized by the left gastric artery arising independently from, and just superior to, a hepatosplenic trunk. This variation, referred to as Type II, was noted by Chen et al. ${ }^{[2]}$ in $4.3 \%$ of the cadavers examined. Variant Type III is characterized by a left gastric artery arising independently from the abdominal aorta and the hepatosplenic trunk arising from a trunk common with the superior mesenteric artery, with the common vessel referred to as a hepatosplenomesenteric trunk. This rare arrangement was seen in only 7 of 974 cadavers $(0.7 \%)$. The remaining variants are classified as Type IV (a single, common celiacomesenteric trunk; 0.7\%), Type V (a common trunk for the left gastric artery and splenic artery and a common trunk for the common hepatic artery and superior mesenteric artery; $1.5 \%$ ) and Type VI (a common trunk for the left gastric and splenic arteries and a common hepatic artery arising from the superior mesenteric artery that travels posterior to the portal vein; $1.8 \%$ ). Here we report a case of a hepatosplenomesenteric trunk (variant Type III) combined with a hepatocolic trunk featuring an accessory right hepatic artery that travels posterior to the portal vein and a wandering mesenteric artery, a combination that is particularly rare. The aim of the study is to raise awareness of vascular anomalies that may have profound clinical consequences. 


\section{Case Report}

As part of a dissection-based course in medical gross anatomy an example of a hepatosplenomesenteric trunk was discovered. The cadaver was a 95-year-old femalewho died from urosepsis and who suffered from chronic anticoagulation, cerebrovascular disease and valvular heart disease. Due to university policies intended to protect donor anonymity, no additional information about the donor is known.

The donor's left gastric artery arises from the anterior surface of the abdominal aorta approximately $1 \mathrm{~cm}$ inferior to the diaphragm at the general location of a typical celiac trunk (Figure 1a). An inferior phrenic artery branches from the left gastric artery $1.7 \mathrm{~cm}$ from the abdominal aorta. The left gastric artery follows the course of the lesser curvature of the stomach where it forms an anastomosis with the right gastric artery. A hepatosplenomesenteric trunk arises from the anterior surface of the abdominal aorta $1.3 \mathrm{~cm}$ inferior to the left gastric artery (Figures 1a and b). Its first branch is a hepatosplenic trunk that arises $5.4 \mathrm{~cm}$ from the origin of the hepatosplenomesenteric trunk. This trunk for the common hepatic artery and splenic artery is $1.5 \mathrm{~cm}$ in length. The splenic artery is tortuous and gives rise to two branches, each $5.3 \mathrm{~cm}$ from the splenic artery's origin, which eventually meet and form a superior polar artery; the superior polar artery is the source of both short gastric arteries and a branch to the abdominal esophagus. Distal to the arteries that form the superior polar artery and $10.8 \mathrm{~cm}$ from its origin at the hepatosplenic trunk the splenic artery gives rise to the left gastroepiploic artery, which forms an anastomosis with the right gastroepiploic artery along the greater curvature of the stomach. The left and right hepatic arteries arise from the common hepatic artery $3.5 \mathrm{~cm}$ from its origin from the hepatosplenic trunk. The right hepatic artery is the source of a cystic artery, the right gastric artery and the gastroduodenal artery. A branch arises from the right margin of the superior mesenteric artery just $3 \mathrm{~mm}$ inferior to the hepatosplenic trunk (Figure 1c). This vessel branches 4 $\mathrm{mm}$ from its origin into an accessory right hepatic artery that passes posterior to the portal vein and an artery that joins with a branch of the left colic artery, i.e., a meandering mesenteric artery, also known as an arc of Riolan. This variant is referred to as a hepatocolic trunk. ${ }^{[3]}$ Near the gall bladder another cystic artery arises from the accessory right hepatic artery. These variations are illustrated schematically in Figure 1d.

\section{Discussion}

Variations in the celiac axis are well studied, especially in regards to the origins and courses of the cystic and hepat- ic arteries. In addition to the system used to characterize these variations proposed by Adachi, others including Varotti et al. ${ }^{[4]}$ have proposed classification systems or have proposed modifications to older systems. Using the system of Varotti et al. ${ }^{[4]}$ the variant in the current study would best fall under Type 3 a as the accessory right hepatic artery is derived from superior mesenteric artery. However, in Varotti's system the right hepatic artery is ultimately derived from a traditional celiac trunk and not from a hepatosplenomesenteric trunk.

Panagouli et al. ${ }^{[5]}$ recently completed a comprehensive survey of studies and case reports involving variations of the celiac trunk. They found the hepatosplenomesenteric trunk to be one of the rarest variants, present in only 49 of 12,196 cases $(0.4 \%)$. According to Chen et al. ${ }^{[2]}$ there are only four case reports based on cadaveric dissection describing a hepatosplenomesenteric trunk: three are described by Adachi and one is in a report by Sekiya et al. ${ }^{[6]}$ In the latter study, the left gastric artery is the source of both an inferior phrenic artery and the left hepatic artery. Unfortunately, details of the branches of the hepatosplenomesenteric trunk are not provided, ${ }^{[6]}$ but it is interesting to note that the cadaver they studied had a number of other vascular variations including a persistent sciatic artery, a meandering mesenteric artery, accessory renal arteries and a retroesophageal right subclavian artery. Of these additional variations only a meandering mesenteric artery, which in our case was derived from the hepatocolic trunk, was observed. Thus, a hepatosplenomesenteric artery can be present in individuals with otherwise typical vasculature outside the superior aspect of the abdomen. Since the review of the literature by Chen et al. ${ }^{[2]}$ hepatosplenomesenteric trunks were reported in a patient undergoing surgery to repair a splenic artery aneurysm ${ }^{[7]}$ and in a patient using computerized tomography. ${ }^{[8]} \mathrm{A}$ recently published cadaveric study describes a phrenicogastric trunk arising proximal to the hepatosplenomesenteric trunk, ${ }^{[9]}$ as do we.

The cadaver examined in our study shows variant vasculature throughout the field of supply of a typical celiac trunk, including a superior polar splenic artery with esophageal branches and an accessory right hepatic artery arising independently from the superior mesenteric artery that travels posterior to the portal vein. This artery is also the source of a dorsal pancreatic artery and the wandering mesenteric artery. Considered individually these additional variations are relatively common. Superior polar arteries have been reported in as many as $51 \%$ of cadavers examined, ${ }^{[10]}$ but to the best of our knowledge this artery has not been reported previously to be the source of esophageal branches. Accessory right 

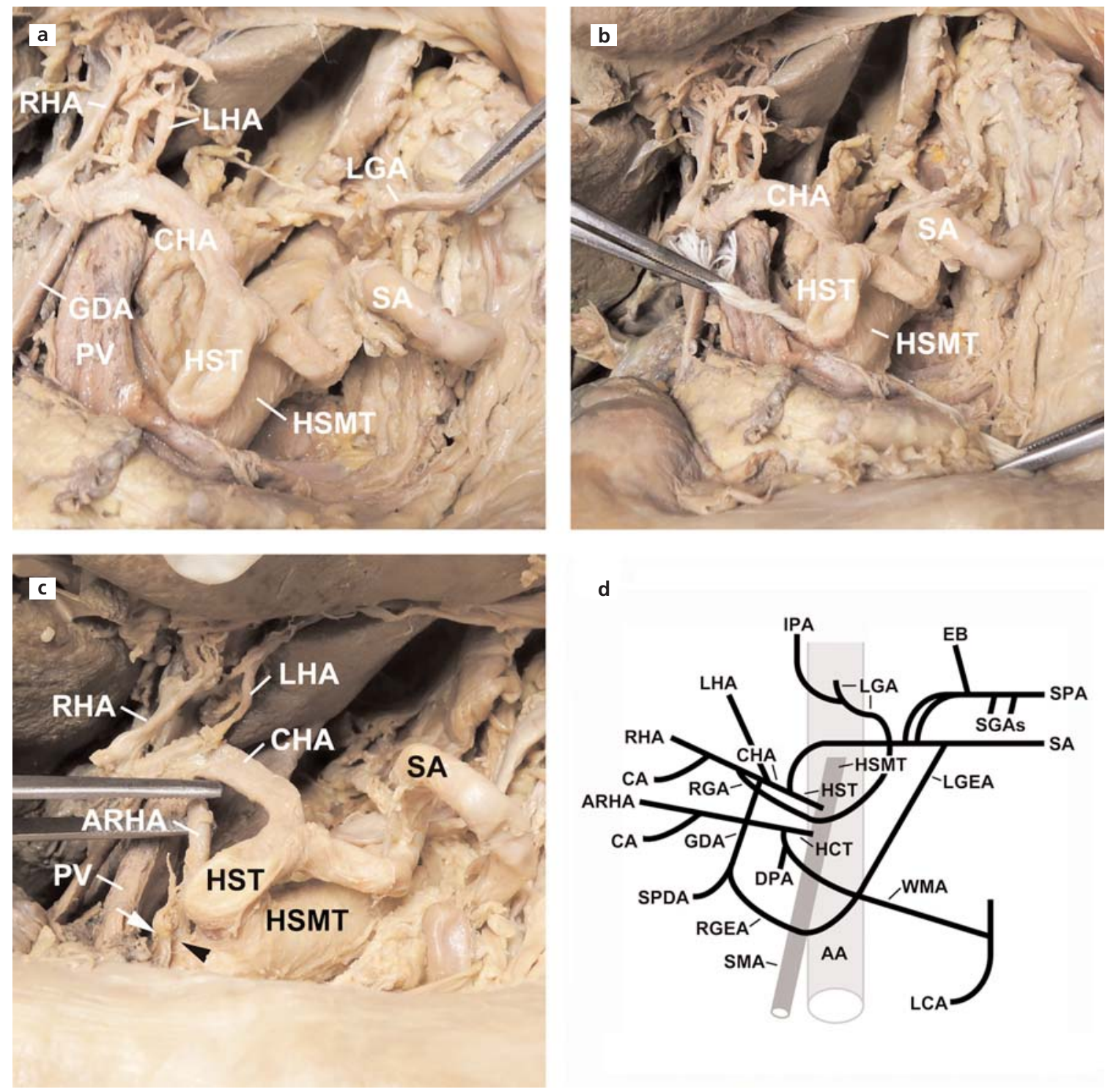

Figure 1. A hepatosplenomesenteric trunk. (a) The left gastric artery (LGA), held by forceps, arises independently from the abdominal aorta. The hepatosplenomesenteric trunk (HSMT) gives rise to a hepatosplenic trunk (HST), which is the source of the splenic artery (SA) and common hepatic artery (CHA). GDA: gastroduodenal artery; LHA: left hepatic artery; PV: portal vein; RHA: right hepatic artery. (b) The HSMT is demonstrated by passing a string between the trunk and the abdominal aorta. (c) A hepatocolic artery (black arrowhead) arises from the superior mesenteric artery approximately $3 \mathrm{~mm}$ inferior to the HST. It is the source of an accessory right hepatic artery (ARHA) that passes posterior to the PV, and a wandering mesenteric artery (white arrow). (d) A schematic drawing illustrating the branches of the HSMT. AA: abdominal aorta; CA: cystic artery; DPA: dorsal pancreatic artery; EB: esophageal branch; HCT: hepatocolic trunk; IPA: inferior phrenic artery; LCA: left colic artery; LGEA: left gastroepiploic artery; RGA: right gastric artery; RGEA: right gastroepiploic artery; SGAs: superior gastric arteries; SMA: superior mesenteric artery; SPA: superior polar artery; SPDA: superior pancreaticoduodenal artery; WMA: wandering mesenteric artery. [Color figure can be viewed in the online issue, which is available at www.anatomy. org.tr]

hepatic arteries arising directly from the superior mesenteric artery are seen in $5 \%$ of cadavers. ${ }^{[2]}$ When it is the source of the dorsal pancreatic artery, as it was in the current study, this vessel typically courses posterior to the portal vein. ${ }^{[1]}$ Wandering mesenteric arteries are present in $10 \%$ of the population. ${ }^{[3]}$
In the embryo, the abdominal aorta is a paired structure connected with a series of channels to ventrally and laterally located splanchnic arteries that supply the developing viscera. As the aortas fuse these channels typically regress, leaving three arteries -the celiac trunk, the superior mesenteric artery and the inferior mesenteric artery- 
supplying the viscera of the foregut, midgut and hindgut, respectively. The variations in this blood supply described by others as well as the series of variations reported here are likely to be the results of the atypical regression and selective retention of the channels connecting the developing aorta with the embryonic splanchnic arteries. ${ }^{[12,13]}$

Though rare, surgeons and radiologists should be aware of the potential for their patients to have a hepatosplenomesenteric trunk, which can be present both in patients with ${ }^{[6]}$ or without (the current case) significant vascular variations outside the abdomen. Failure to recognize the presence of such variations can result in complications following organ transplantation, gastrointestinal procedures and the surgical excision of tumors, as well as the inadequate embolization of unexpected vessels by interventional radiologists.

\section{Acknowledgements}

S.E.J., D.D.O, A.E.S. and V.S.M. contributed equally to this study. The authors are grateful to staff of the University of California at Davis Body Donation Program and wish to thank the individuals who donated their bodies and tissues for the advancement of education and research. The authors declare that they have no conflict of interest.

\section{References}

1. Haller AV. Icones anatomicae in quibus aliquae partes corporis humani delineatae proponuntur et arteriarum potissimum historia continetur. Göttingen: A. Vandenhoeck; 1756.

2. Chen H, Yano R, Emura S, Shoumura S. Anatomic variation of the celiac trunk with special reference to hepatic artery patterns. Ann Anat 2009;191:399-407.
3. Michels NA, Siddharth P, Kornblith PL, Parke WW. The variant blood supply to the descending colon, rectosigmoid and rectum based on 400 dissections. Its importance in regional resections: a review of medical literature. Dis Colon Rectum 1965;8:251-78.

4. Varotti G, Gondolesi GE, Goldman J, Wayne M, Florman SS, Schwartz ME, Miller CM, Sukru E. Anatomic variations in right liver living donors. J Am Coll Surg 2004;198:577-82.

5. Panagouli E, Venieratos D, Lolis E, Skandalakis P. Variations in the anatomy of the celiac trunk: a systematic review and clinical implications. Ann Anat 2013;195:501-11.

6. Sekiya S, Horiguchi M, Komatsu H, Kowada S, Yokoyama S, Yoshida K, Isogai S, Nakano M, Koizumi M. Persistent primitive sciatic artery associated with other various anomalies of vessels. Acta Anat (Basel) 1997;158:143-9.

7. Sakakibara K, Shindo S, Matsumoto M, Yoshida Y, Kimura M, Honda Y, Kamiya K, Katsu M, Kaga S, Suzuki S. Splenic artery aneurysm of the hepatosplenomesenteric trunk. Ann Vasc Dis 2013; 6:730-3.

8. Maldjian PD, Chorney MA. Celiomesenteric and hepatosplenomesenteric trunks: characterization of two rare vascular anomalies with CT. Abdom Imaging 2015;40:1800-7.

9. Prasanna LC, Alva R, Sneha GK, Bhat KM. Rare variations in the origin, branching pattern and course of the celiac trunk: report of two cases. Malays J Med Sci 2016;23:77-81.

10. Sahni DA, Jit IB, Gupta CN, Gupta DM, Harjeet E. Branches of the splenic artery and splenic arterial segments. Clin Anat 2003;16:3717.

11. Matsumura H. The significance of the morphology of the dorsal pancreatic artery in determining the presence of the accessory right hepatic artery passing behind the portal vein. Kaibogaku Zasshi 1998;73:517-27.

12. Tandler J. Über die varietäten der Arteria coeliaca und deren Entwicklung. Anat Hefte 1904;25:473-500.

13. Cavdar S, Sehirli U, Pekin B. Celiacomesenteric trunk. Clin Anat 1997;10:231-4.

\footnotetext{
This is an open access article distributed under the terms of the Creative Commons Attribution-NonCommercial-NoDerivs 3.0 Unported (CC BY-NCND3.0) Licence (http://creativecommons.org/licenses/by-nc-nd/3.0/) which permits unrestricted noncommercial use, distribution, and reproduction in any medium, provided the original work is properly cited. Please cite this article as: Johnson SE, Odineal DD, Steele AE, Stone VM, Tucker RP. A case of a hepatosplenomesenteric trunk combined with a hepatocolic trunk. Anatomy 2017;11(1):46-49.
} 